\title{
Bio-inspired Obstacle Avoidance: From Animals to Intelligent Agents
}

\author{
Ruben Nuredini ${ }^{1}{ }^{*}$, Bekim Fetaji ${ }^{2}$, Ivan Chorbev $^{3}$ \\ ${ }^{1}$ Department for Software Engineering, Heilbronn University of Applied Sciences, Heilbronn, Germany. \\ ${ }^{2}$ Department for Contemporary Sciences and Technologies, South-East European University, Tetovo, \\ Macedonia. \\ ${ }^{3}$ Faculty of Computer Science and Engineering, Ss. Cyril and Methodius University, Skopje, Macedonia. \\ * Corresponding author. Tel.: +49 7131504 236; email: ruben.nuredini@hs-heilbronn.de \\ Manuscript submitted November 8, 2016; accepted May 9, 2017. \\ doi: $10.17706 /$ jcp.13.2.146-153
}

\begin{abstract}
A considerable amount of research in the field of modern robotics deals with mobile agents and their autonomous operation in unstructured, dynamic, and unpredictable environments. Designing robust controllers that map sensory input to action in order to avoid obstacles remains a challenging task. Several biological concepts are amenable to autonomous navigation and reactive obstacle avoidance. We present an overview of most noteworthy, elaborated, and interesting biologically-inspired approaches for solving the obstacle avoidance problem. We categorize these approaches into three groups: nature inspired optimization, reinforcement learning, and biorobotics. We emphasize the advantages and highlight potential drawbacks of each approach. We also identify the benefits of using biological principles in artificial intelligence in various research areas.
\end{abstract}

Key words: Autonomous navigation, obstacle avoidance, biologically-inspired learning.

\section{Introduction}

Developing a reliable navigation system for mobile agents is an attractive topic causing the area of mobile robotics to attract vast amount of researchers. The emphasis in today's mobile agent development is set on the concept of autonomy - the property of a system that can change its behavior in response to unanticipated events during their operation. When observed from a general perspective, the autonomous navigation task can be decomposed into several subtasks: localization, mapping, and path planning. Obstacle avoidance is a subtask of path planning that arises when the agent operates in dynamic environments and unpredictable events. It relies on on-line sensory information and the appropriate action in order to prevent collisions and real-time operation and reactivity are the key properties that such system should possess.

Natural evolution caused remarkable biological phenomena that resulted with exhibition of outstanding abilities at almost every level of the animal world. Simple animals display behaviors that are goal-oriented, adaptive, opportunistic, plastic, and robust - qualities that are considered to be essential for every modern artificial system. Moreover, they display agile and versatile locomotion abilities. Such observations have been inspiration for many researchers to work on developing biologically-inspired control strategies for path planning and obstacle avoidance.

This paper focuses on biologically-inspired methods for solving the path planning and obstacle avoidance tasks. The next section deals with nature-inspired optimization techniques such as genetic algorithms and 
swarm intelligence and the recent publications involving their usage in solving the path planning problem. In Section 3 we focus on reinforcement learning as a nature inspired paradigm and its suitability for obstacle avoidance. Section 4 deals with biorobotics and employing biological principles in robotic applications. We conclude by summarizing all three approaches identifying their advantages and drawbacks.

\section{Nature Inspired Optimization Approaches}

In particular cases, the solution of the obstacle avoidance problem is included in the solution of the global path planning problem. This occurs when: a) the full map of a structured and static environment is available to the agent; or b) the dynamics of the environment (trajectory of the moving obstacles and other agents) are perfectly known a priori. Dynamic obstacles are treated as static and re-planning is necessary whenever changes are registered. The solution of the global path planning problem usually reduces to the selection of the best possible trajectory with regard to one or a combination of several criteria. This property of the problem makes it eligible for the application of optimization techniques. Due to the subject of this paper, we focus only on the bio-inspired optimization algorithms.

Genetic algorithms (GA) are adaptive heuristic search algorithms that are used to solve optimization problems by exploiting historical information to direct the search into the region of better performance within the search space. The fact that in path planning the main concern is finding the optimal out of many possible trajectories, finding the best trajectory can be observed as optimization problem.

In [1] the authors presented how application of multi-objective GA deals with several issues for optimization of two dimensional path planning. They experimented with several path representation schemes, different number of optimal path criteria (path length, path vulnerability, and path smoothness) and compared the performance results with some of the existing similar approaches. In [2] GA techniques are used for improvement of the randomly selected paths between the start and the goal position for a mobile robot in outdoor environments. In the study presented in [3] a new and improved mutation operator for the GA is applied to the path planning problem. The improved mutation method simultaneously checks all free nodes close to the mutation node and accepts the node according to the fitness value of the total path. GA have also been used for multi-agent path planning optimization in static environment [4] as well as for global path planning and local path refinement [5].

Particle Swarm Optimization (PSO) [6] is a population based stochastic optimization technique inspired by the social behavior and adaptation capability of biological populations such as flocks of birds, schools of fishes, and animal herds. PSO is similar to GA since it generates a set of random solutions (the initial swarm) in the design space and then the potential solutions (particles) propagate towards the optimal solution over a number of iterations (moves). Early successful application of PSO for global path planning was presented in [7] where PSO is employed for optimizing the shortest path in a MAKLINK graph obtained by the Dijkstra's algorithm. In [8] the authors studied the path planning problem in environments with precise positions of danger sources by employing multi-objective PSO. Other publications include: path planning by introducing chaos in the PSO method [9]; employing PSO for optimization of a Cartesian point-to-point path [10]; fitness-scaling adaptive chaotic PSO for path planning for UCAV with performance comparison to other methods [11]; employing a PSO technique for optimizing the parameters of a path represented by string of cubic splines [12]; as well as a study of four variants of PSO performance on path planning and obstacle avoidance of a robotic arm [13].

Ant Colony Optimization (ACO) is a probabilistic technique for solving computational problems inspired by the foraging behavior of ants. Ants' capability for finding the shortest path to the food source is used for solving problems that can be reduced to finding feasible and optimal paths through graphs. Recent publications present several examples of ACO techniques used for solving the path planning problem. In [14] 
the authors present an improved ACO algorithm entitled Heterogeneous Feature ACO. Here they define two types of ants specialized in finding random paths and optimize them as well as a method for adjusting the number of the ants of each type. The authors of [15] present an algorithm that combines MAKLINK graph theory, Dijkstra algorithm, and ACO in a three-step real-time globally optimal path planning algorithm.

Other recent and noteworthy examples for application of bio-inspired optimization algorithms for solving the global path planning problem are: artificial bee colony based algorithm for solving a problem similar to the travelling salesperson problem [16] and employing the pigeon-inspired optimization for solving the air robot path planning problem [17].

\section{Reinforcement Learning Approaches}

Reinforcement learning (RL) is a sub-domain of machine learning that deals with solving sequential decision tasks through trial and error interactions with the environment. The agents make their choice of actions toward maximizing some notion of a long-time reward. It is tightly related to the psychology of animal behavior as it represents a good model for instrumental learning. The center of all RL systems is the policy that maps the states of the world with agent actions. The RL algorithm gradually tunes the policy based on the obtained reward for every interaction of the agent with the environment. As the agent gathers experience of its actions and the associated reward, the system will eventually find the optimal policy. RL systems stand out because of their ability to self-learn the appropriate action for a state instead of the classical approach of explicitly predefining how the task should be achieved. These systems quickly adapt the agent's behavior on changes in the surrounding and therefore generalize well on every environment.

The idea of a behavioral controller obtained by interaction with the environment motivated many researchers to apply this concept toward solving the obstacle avoidance task. By defining a suitable reward function relevant to the task a stimulus-response controller can spontaneously emerge as a mapping of the perceived state and the desirable action.

In an early attempt, a reinforcement connectionist learning system was developed in order to find safe paths for a mobile robot in a "non-maze-like" environment [18]. The input to the system was the attractive and repulsive forces exerted by the goal and the obstacles, and its output was the robot action described by the step length and the orientation. The robot moved in response to the sensorial information is assessed by a reinforcement signal in form of a real number representation of the evaluation criterion - a compromise between minimizing path length and maximizing the distance to the obstacles and walls.

There are several efforts of using the Q-learning reinforcement algorithm for the problem of reactive obstacle avoidance in indoor environments. A combined RL system with two behaviors (a goal-driven and reflexive) was presented in [19]. The agent separately learned both behaviors - the goal-driven (shooting a ball in a goal), and the reactive (avoiding obstacles. The state was represented by simplification of the visual information of the position of the ball and the goal whereas the action set were the discretized commands to the motors. Two methods of coordination of both behaviors were proposed: simple summation of the values of the two Q-functions, and switching the Q-functions according to a carefully designed decision rule.

In [20] the agent's state was determined by an array of infrared sensors and the greedy strategy was used to select one of the possible actions to move and rotate. In order to overcome the problem caused by the enormous number of state-action pairs a feed-forward neural network was used to store the Q-values. In the initial stages of learning, the selected actions caused numerous collisions with the obstacles. After extensive training, the performance significant improved and the number of collisions decreased.

Similar approach of using Q-learning with neural networks is recorded in [21]. In order to improve the convergence rate of the control network, the input space was discretized in clusters by employing a Kohonen neural network structure with the winner-take-all unsupervised learning rule. The approach was verified in 
simulation environment where the mobile agent developed obstacle avoidance capability out of initially unknown control strategy.

All aforementioned applications face similar problem since they have to cope with realistic physical systems. The state spaces in such environment are continuous and high-dimensional causing the "the curse of dimensionality". This problem is inherent to RL and leads to vast amount of memory and computational requirements in order to cover the state spaces. Typical measures to overcome "the curse" are discretization of the state space, and function approximation of the Q-function. Most applications employ both. The discretization should be well defined or otherwise the system can suffer from insufficient representation which can affect the learning process.

In a very elaborative study obstacle avoidance method based on monocular visual cues and RL was achieved by discretization of the real-world image [22]. In the first step, image pre-processing techniques such as segmentation and color transformation were applied to both, realistic and synthetic images in order to obtain the feature vector. This vector along with the labeled data was the used to learn (using supervised learning) to estimate the distances to objects in an image and therefore produce the output of the vision system. Finally, a policy search RL approach was employed in order to develop a controller that associates the output of the vision system with steering commands.

\section{Biorobotic Approaches}

The scientific field of bio-inspired robotics (biorobotics, biomimetics) deals with applying biologically plausible principles to autonomous robots. In general, this means building computational models of individual biological features and behaviors as well as physiological and evolutionary processes of humans and simpler natural animals.

There is a high level of research activity on obstacle-avoidance behavior of primitive animals from behavioral and evolutional perspective. The main inspiration of these studies is the fact their limited-size brains and simple nervous systems display immense performance in tasks such as guidance and navigation. The real-time perception as well as the reactive senso-motoric response in uncontrolled environments has been the motivation behind the attempts to create a behavioral controller that maps visual stimulus to actions.

One of the earliest models that employs biological principles for solving the autonomous navigation and obstacle avoidance task was inspired by the earthworm's simple navigation system [23]. Simple navigation routines were hierarchically organized and linked by failure defaults. The succeedingly more difficult problems would invoke more sophisticated routines only on failure of the lower levels causing problems to never propagate at a level higher than the one needed for their solution. The proposed algorithm did not require object identification and strategic planning and was therefore independent form the sensor type.

In another similar study [24] the autonomous navigation problem is addressed from perspective of the subsumption architecture - a layered approach of autonomous control in which advanced task-achieving behaviors can be achieved by combining simple tasks from lower levels [25]. This effort illustrates how new forms of behavioral patterns emerge when different primitive reflexive behaviors of an autonomous agent are allowed to operate concurrently. The idea was based on the accepted claim in the theory of animal behavior - animals possess inherited responses to certain situations and combine them with the ability to adapt to situations for which no response is available. Autonomy was developed from a set of simple forms of primitive reflexive behaviors modeled as a simple stimulus/response mechanism and classified into repulsive and attractive. Concurrent activation of a set of primitive reflexive behaviors in a simulation and actual mobile robot resulted with emergent behaviors such as wandering, simple navigation, perimeter following etc. 
Some of the most interesting cases of bio-inspired behavior modeling builds on the neurobiological assumption that perception and action (visuomotoric) are a coupled system that has to be investigated in a closed loop [26], [27]. In [27] obstacle avoidance was achieved through simultaneous adaptation of viewing direction of the sensors and the sensorimotor coupling. The agent was equipped photoreceptors and motors and the visual input was processed by motion detectors that are further coupled via transmission weights to the motors. The weighted connection between sensory input and motor output were optimized by genetic algorithm. Another line of work explores the concept of artificial evolution for development of behavioral abilities without any constrains on the architecture and functioning modalities [28]. Visual information was used as an input to a network of spiking neurons and genetic algorithms were used to evolve it. This resulted with a spiking controller capable to perform the navigation and obstacle avoidance task of an autonomous agent in lab environment.

Recently, several strategies for obstacle avoidance inspired by features of insects' visual systems have been presented based on mapping monocular optic flow into control signals [29], [30], and depth estimation from 3D reconstruction of images acquired by a modular stereo-vision [31].

\section{Conclusion}

Taking biology as inspiration for artificial intelligence has become usual practice. We identified three biologically inspired approaches for creating autonomous behavior of intelligent agents with focus is on the obstacle-avoidance task of mobile agents.

In cases when the full model of the operating environment is available to the agent, the obstacle avoidance task is encompassed into the more general path planning task. Path planning can be observed as an optimization task making and there are numerous examples of usage of nature-inspired optimization techniques for solving it.

Recent progress in the field of neuroscience takes us closer to the discovery of how human brain works. It is still a mystery though what is the relationship between the brain and the mind. On the other side, several learning paradigms are well established in the literature. One of them - the instrumental conditioning occurs by successively shaping animals' behavior by gradual approximation based on strategically and well-timed reinforcement. Reinforcement learning is a well-established machine learning technique that is based on instrumental conditioning. The idea of achieving obstacle avoidance behavior of mobile agents by letting it interact with the environment motivated many researchers. Several problems caused by intractable state spaces, and undermodeling in simulations are yet to be resolved.

Many robots capable of acting in the real world are largely based on biological principles such as physiological processes and evolution. Several attempts have been presented that aim to achieve obstacle avoidance behavior by designing controllers that link agents' sensors and actuators resembling animals' stimulus-response mechanism in the senso-motoric loop. Yet current robotic and biological systems have obvious difference in the exhibited behavior. By comparing and analyzing them it becomes clear that the limitation of the robotic systems is mainly due to the still bounded information processing capabilities. This observation justifies the claim that low-level senso-motorical (contrary to analytical) skills typical for living beings require enormous computational power.

Despite all, simulation of biological principles brings benefit to multiple scientific disciplines. The evolutionary development of non-trivial biological processes offers an inspirational starting point for artificial intelligence research. This can help identify issues related to these processes and spark discussions and investigation in the field of biology. On the other hand, simulation environments can serve as a playground for examination and evaluation of biological models. The primary motivation for such experiments is to find answers to question about the structure and function of the animal brains as well as 
the evolutionary and ontogenetic processes. However, they usually yield insights for potential application in engineering as a secondary benefit.

\section{References}

[1] Ahmed, F., \& Deb, K. (2013). Multi-objective optimal path planning using elitist non-dominated sorting genetic algorithms. Soft Computing, 17(7), 1283-1299.

[2] Tarokh, M. (2008). Hybrid intelligent path planning for articulated rovers in rough terrain. Fuzzy Sets and Systems, 159(21), 2927-2937.

[3] Tuncer, A., \& Yildirim, M. (2011). Dynamic path planning of mobile robots with improved genetic algorithm. Computers and Electrical Engineering, 23(6), 1564-1572.

[4] Kala, R. (2012). Multi-robot path planning using co-evolutionary genetic programming. Expert Systems with Applications, 39(3), 3817-3831.

[5] Zhu, Z., Wang, F., He, S., \& Sun, Y. (2015). Global path planning of mobile robots using a memetic algorithm. International Journal of Systems Science, 46(11), 1982-1993.

[6] Kennedy, J., \& Eberhart, R. (1995). Particle swarm optimization. Proceedings of IEEE International Conference on Neural Networks IV.

[7] Qin, Y. Q., Sun, D. B., Li, N., \& Cen, Y. G. (2004). Path planning for mobile robot using the particle swarm optimization with mutation operator. Proceedings of International Conference on Machine Learning and Cybernetics.

[8] Gong, D.-W., Zhang, J.-H., \& Zhang, Y. (2011). Multi-objective particle swarm optimization for robot path planning in environment with danger sources. Journal of Computers, 6(8), 1554-1561.

[9] Zhao, Q., \& Yan, S. (2005). Collision-free path planning for mobile robots using chaotic particle swarm optimization. Proceedings of the 1st International Conference on Advances in Natural Computation.

[10] Xu, W., Li, C., Llang, B., Liu, Y., \& Xu, Y. (2008). The cartesian path planning of free-floating space robot using particle swarm optimization. Journal of Advanced Robotic Systems, 5(3), 301-310.

[11] Zhang, Y., Wu, L., \& Wang, S. (2013). UCAV path planning by fitness-scaling adaptive chaotic particle swarm optimization. Mathematical Problems in Engineering.

[12] Saska, M., Macas, M., Preucil, L., \& Lhotska, L. (2006). Robot path planning using particle swarm optimization of ferguson splines. Proceedings of the 11th IEEE International Conference on Emerging Technologies and Factory Automatio. Prague: Czech Republic.

[13] Chyan, G. S., \& Ponnambalam, S. G. (2012). Obstacle avoidance control of redundant robots using variants of particle swarm optimization. Robotics and Computer-Integrated Manufacturing, 28(2), 147-153.

[14] Yao, Y., Ni, Q., Lv, Q., \& Huang, K. (2015). A novel heterogeneous feature ant colony optimization and its application on robot path planning. Proceedings of IEEE Congress on Evolutionary Computation. Japan: Sendaj.

[15] Tan, G.-Z., He, H., \& Sloman, A. (2007). Ant colony system algorithm for real-time globally optimal path planning of mobile robots. Acta Automatica Sinica, 33(3), 279-285.

[16] Chang, W. L., Zeng, D., Chen, R.-C., \& Guo, S. (2013). An artificial bee colony algorithm for data collection path planning in sparse wireless sensor networks. Journal of Machine Learning and Cybernetics, 6(3), 375-383.

[17] Duan, H., \& Qiao, P. (2014). Pigeon-inspired optimization: A new swarm intelligence optimizer for air robot path planning. Journal of Intelligent Computing and Cybernetics, 7(1), 24-37.

[18] Del Millán, J. R., \& Torras, C. (1992). A reinforcement connectionist approach to robot path finding in non-maze-like environments. Reinforcement Learning, 139-171. US: Springer. 
[19] Asada, M., Uchibe, E., Noda, S., Tawaratsumida, S., \& Hosoda, K. (1994). Coordination of multiple behaviors acquired by a vision-based reinforcement learning. Proceedings of the IEEE/RSJ/GI Conference on Intelligent Robots and Systems. Germany: Munich.

[20] Huang, B.-Q., Cao, G.-Y., \& Guo, M. (2005). Reinforcement learning neural network to the problem of autonomous mobile robot obstacle avoidance. Proceedings of the International Conference on Machine Learning and Cybernetics.

[21] Macek, K., Petrovic, I., \& Peric, N. (2002). A reinforcement learning approach to obstacle avoidance of mobile robots. International Workshop on Advanced Motion Control.

[22] Michels, J., Saxena, A., \& Ng, A. Y. (2005). High speed obstacle avoidance using monocular vision and reinforcement learning. Proceedings of International Conference on Machine Learning.

[23] Cahn, D. F., \& Phillips, S. R. (1975). ROBNAV: A range-based robot navigation and obstacle avoidance algorithm. IEEE Trans. Systems, Man, and Cybernetics, 5, 544-551.

[24] Anderson, T. L., \& Donath, M. (1990). Animal behavior as a paradigm for developing robot autonomy. Robotics and Autonomous System, 6(1-2), 145-168.

[25] Brooks, R. A. (1986). A robust layered control system for a mobile robot. IEEE Journal of Robotics and Automation, 2(1), 14-23.

[26] Reynolds, C. W. (1993). An evolved, vision-ba sed model of obstacle avoidance behavior, from animals to animats 2. Proceedings of the 2nd International Conference on Simulation of Adaptive Behavior.

[27] Neumann, T. R., Huber, S. A., \& Bülthoff, H. (1997). Minimalistic approach to 3D obstacle avoidance behavior from simulated evolution. Proceedings of the Conference on Artificial Neural Networks, Lausanne.

[28] Floreano, D., Epars, Y., Zufferey, J.-C., \& Mattuissi, C. (2006). Evolution of spiking neural circuits in autonomous mobile robots. International Journal of Intelligent Systems, 21, 1005-1024.

[29] Beyeler, A., Zufferey, J.-C., \& Floreano, D. (2009). Vision-based control of near-obstacle flight. Autonomous Robots, 27(201), 201-219.

[30] Milde, M. B., Bertrand, O., Benosman, R., Egelhaaf, M., \& Chicca, E. (2015). Bioinspired Event-Driven Collision Avoidance Algorithm Based on Optic Flow. Proceedings of the Conference on Event-based Control, Communication, and Signal Processing. Poland: Krakow.

[31] Chessa, M., Murgia, S., Nardelli, L., Sabatini, S. P., \& Solari, F. (2014). Bio-inspired active vision for obstacle avoidance. Proceedings of the Conference on Computer Graphics Theory and Applications, Lisbon.

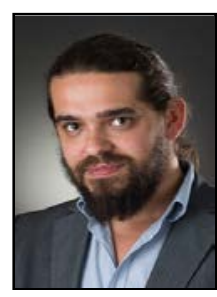

Ruben Nuredini obtained his master of information \& communication technologies at the Faculty of Information \& Communication Technologies, FON University in Skopje in 2011. He earned his bachelor degree at the Faculty for Computer Sciences and Technologies in Tetovo in 2007. Currently he works as a teacher and research associate on the Faculty for Informatics, Heilbronn University, Heilbronn, Germany. Currently he is active in research related to his $\mathrm{PhD}$ thesis in the field of intelligent mobile autonomy. Other research interest fields include machine learning, intelligent systems, parallel programming, application of computer science in robotics and mobile systems, computational neuroscience, data mining. He has published several research papers in international conferences and journals.

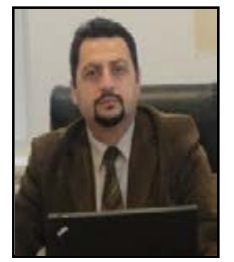

Bekim Fetaji is an Associate Professor. He is a doctor of computer sciences in Graz University of Technology, Austria. He was also the master of sciences in computing at Oxford, England, UK. in 2004. Currently he is employed in Computer Sciences Department in South East European University in Macedonia. He was the best high school student of the generation in 1993, 
Gymnasium Pance Popovski, Gostivar Macedonia. He obtained the best paper award in International Computer Science Symposium at Saga University in 2009. He received the tempus individual research project grant for 1 month in June 2007 - Graz University of Technology, Austria. Currently, he has published more than 90 research papers in several distinguished conferences among which 24 papers in ACM and IEEE conferences, 27 in International Journals and 3 in Journals with ISI web of Science Impact Factor. He was a member of the IEEE and the IEEE Computer Society 2006-2008, 2010-2011, 2013-2014.

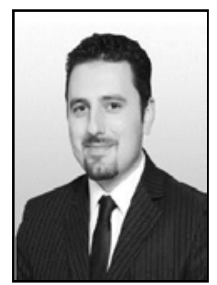

Ivan Chorbev obtained his $\mathrm{PhD}$, doctor of computer sciences at the Faculty of Electrical Engineering and Information Technologies, University Ss. Cyril and Methodius in Skopje in 2009. He earned his bachelor and master degrees at the Faculty of Electrical Engineering in Skopje in 2004 and 2006. Currently he is the dean of the Faculty of Computer Science and Engineering, UKIM, Macedonia. Research post-doc mobility at the Technical University in Viena. The fields of his research interests include combinatorial optimization, heuristic algorithms, constraint programming, application of computer science in medicine and telemedicine, assistive technologies, knowledge extraction, machine learning. He has published more than 80 research papers in several conferences, journals, books. He is a member of the IEEE and the IEEE Computer Society. 